\title{
Physiological Falls Risk Assessment in Older People with Alzheimer's Disease
}

\author{
Edwina R. Lorbach ${ }^{\mathrm{a}}$ Kate E. Webster ${ }^{\mathrm{a}}$ Hylton B. Menz ${ }^{\mathrm{a}} \quad$ Joanne E. Wittwer ${ }^{\mathrm{a}}$ \\ John R. Merory ${ }^{b}$ \\ ${ }^{a}$ Musculoskeletal Research Centre, La Trobe University, Bundoora, and ${ }^{\mathrm{b}}$ Medical and Cognitive Research Unit, \\ Neurology Department, Austin Health, Melbourne, Australia
}

\section{Key Words}

Alzheimer's disease $\cdot$ Accidental falls $\cdot$ Dementia $\cdot$ Balance

\begin{abstract}
Background: Falls are common in people with Alzheimer's disease (AD). There is some evidence that deficits in vision, peripheral sensation, strength, reaction time and balance may be partly responsible for this increased risk. Aims: To determine the feasibility and test-retest reliability of a physiological test battery designed to assess falls risk [the Physiological Profile Assessment (PPA)] in people with AD, and to compare their PPA scores to age- and sex-matched controls. Methods: Twenty-one community-dwelling people with probable, mild to moderate AD aged 63-91 years, and 21 age- and sex-matched controls underwent the PPA tests and the Mini-Mental State Examination. All tests were then repeated in the AD group to determine test-retest reliability. Results: Most of the PPA tests could be successfully administered to participants with AD. The AD group had a significantly higher overall falls risk score $\left(\mathrm{t}_{40}=-2.41, \mathrm{p}<0.02\right)$, slower hand $\left(\mathrm{t}_{40}=-4.86, \mathrm{p}<0.01\right)$ and foot reaction time $\left(\mathrm{t}_{40}=-2.26, \mathrm{p}<0.05\right)$ and worse coordinated stability $\left(\mathrm{t}_{40}=\right.$ $-2.40, p<0.05)$ than the controls. Conclusion: Physiological falls risk assessment is feasible in older people with mild to moderate AD. Older people with AD demonstrate significant impairments in several physiological domains, particularly reaction time, compared to controls.
\end{abstract}

Copyright $\odot 2007$ S. Karger AG, Basel

\section{KARGER \\ Fax +41 613061234 \\ E-Mail karger@karger.ch}

(c) 2007 S. Karger AG, Basel

$1420-8008 / 07 / 0244-0260 \$ 23.50 / 0$

www.karger.com

\section{Introduction}

Older people with Alzheimer's disease (AD) experience a higher rate of falls and fall-related injury than cognitively intact older people [1-5]. While the mechanisms contributing to falls in this group are poorly understood, there is some indication that physiological deficits may underlie this increased susceptibility [6]. Physiological risk factors for falls, such as impaired vision [7], slowed reaction time [8] and increased postural sway [9], are more pronounced in people with $\mathrm{AD}$ compared to controls, and people with AD exhibit significantly greater variability in gait patterns $[10,11]$.

Several studies have now confirmed that impairments in sensorimotor function, including reduced vision, peripheral sensory loss, slowed reaction time and muscle weakness, are strongly associated with impaired balance and an increased risk of falling $[12,13]$. One approach for assessing physiological falls risk in older people - the Physiological Profile Assessment (PPA) - has been shown to have $75 \%$ accuracy for predicting falls in older people over a 12-month period [14]. The PPA includes tests of vision, sensation, strength, reaction time and balance, and generates an overall falls risk score based on normative data from population-based samples of older people. The PPA has been shown to be reliable and sensitive to improvements in function associated with exercise [14].

A/Prof. Hylton B. Menz

Musculoskeletal Research Centre, Faculty of Health Sciences

La Trobe University

Bundoora 3086, Vic. (Australia)

Tel. +61 39479 5801, Fax +61 39479 5415, E-Mail h.menz@latrobe.edu.au 
Given previous observations of impaired sensorimotor and balance function in older people with $\mathrm{AD}$, it is likely that the PPA may have some value in assessing falls risk in this population. However, the PPA has not been specifically evaluated in older people with AD. Due to cognitive limitations associated with the condition, the feasibility of undertaking physiological falls risk assessment in people with AD is uncertain. Therefore, the aim of this study was to assess the feasibility and test-retest reliability of the PPA in people with AD, and to compare PPA performances between AD cases and controls.

\section{Methods}

\section{Participants}

The AD group comprised 10 men and 11 women aged 63-91 years (mean \pm standard deviation $=79.3 \pm 6.3$ ) with a diagnosis of probable $\mathrm{AD}$ according to the National Institute of Neurological and Communicative Disorders and Stroke and the Alzheimer's Disease and Related Disorders Association criteria [15]. Participants were consecutively recruited from an outpatient neurology clinic and were included if they had mild to moderate dementia [as determined by a Mini-Mental State Examination (MMSE) score of 11-26 [16]], were 60 years or older, walked independently without a gait aid indoors and were free from significant musculoskeletal, cardiac or neurological impairments. Historical control data were obtained from a sample of age- and sex-matched retirement village residents aged 60-90 years (mean \pm standard deviation $=79.2 \pm 6.0$ ). Participant characteristics are shown in table 1. The University Human Ethics Committee approved the aims and procedure for this study and participants gave written consent.

\section{Study Design}

AD participants underwent the PPA and MMSE on 2 occasions 1 week apart, and reliability coefficients for each were calculated. To control for diurnal variation, participants were tested at the same time of day on each occasion. PPA and MMSE scores in the $\mathrm{AD}$ group (averaged across both measurement sessions) were correlated to investigate associations between cognition and performance on each of the physiological tests. Baseline PPA measurements were then compared to scores from cognitively intact controls.

\section{Apparatus and Procedure}

The PPA

The PPA protocol was followed and is described in detail elsewhere [14]. Briefly, visual acuity was binocularly assessed using letter charts with high- and low-contrast letters and measured in terms of logarithm of the minimum angle resolvable in minutes of arc. Edge contrast sensitivity was binocularly assessed using the Melbourne Edge Test. For vision tests, near-distance or distance glasses were worn where applicable. Knee extension and ankle dorsiflexion strength of the dominant limb were isometrically assessed using a spring gauge. Simple reaction time of the
Table 1. Participant characteristics

\begin{tabular}{lcc}
\hline Characteristic & $\begin{array}{c}\text { AD cases } \\
(\mathrm{n}=21)\end{array}$ & $\begin{array}{c}\text { Controls } \\
(\mathrm{n}=21)\end{array}$ \\
\hline Female:male & $11: 10$ & $11: 10$ \\
Age, years & $79.3 \pm 6.3$ & $79.2 \pm 6.0$ \\
Height, cm & $166 \pm 9$ & $164 \pm 12$ \\
Weight, kg & $68 \pm 10$ & $75 \pm 17$ \\
Education, years & $12 \pm 4$ & $10 \pm 3$ \\
Duration of AD, months & $17 \pm 16$ & - \\
History of falls in previous 12 months & $7(33)$ & $6(29)$ \\
Previous stroke & $3(14)$ & $1(5)$ \\
Diabetes & $2(10)$ & $2(10)$ \\
History of cancer & $1(5)$ & $6(29)^{\mathrm{a}}$ \\
History of heart problems & $5(24)$ & $8(38)$ \\
Hypertension & $14(67)$ & $13(62)$ \\
Incontinence & $1(4.8)$ & $5(24)$ \\
Osteoarthritis & $7(33)$ & $18(86)^{\mathrm{a}}$ \\
Number of medications & $4 \pm 2$ & $5 \pm 3^{\mathrm{a}}$ \\
Currently taking AD medication & $15(71)$ & 0 \\
\hline
\end{tabular}

Figures are numbers of participants with percentages in parentheses.

a Significant difference between AD cases and controls, $\mathrm{p}<0.05$.

dominant hand and foot was measured using an electronic timer with a red light as the stimulus and depression of a switch as the response. Tactile sensitivity was measured using a pressure aesthesiometer placed on the lateral malleolus of the dominant foot. Proprioception was measured using a lower-limb matching task, with errors recorded using a protractor inscribed on a vertical clear acrylic sheet (height $=60 \mathrm{~cm}$, width $=60 \mathrm{~cm}$, depth $=1 \mathrm{~cm}$ ) placed between the legs.

To assess body sway, a rod with a vertically-oriented pen (swaymeter) extended from the waist and recorded the body's displacements with eyes open and closed while standing on the floor and on a $15-\mathrm{cm}$-thick, medium-density foam rubber mat. Near-tandem stability was measured with the sway-meter directed posteriorly, feet separated laterally $(2.5 \mathrm{~cm})$ by standardized markings and with the heel of the right foot $2.5 \mathrm{~cm}$ anterior to the great toe of the back foot. Participants attempted to stand motionless for $30 \mathrm{~s}$ with eyes open and arms clasped in front, and the degree of medio-lateral excursion (millimetres) was measured. The test was terminated when participants took a step or contacted an external surface. Leaning balance was measured using the maximal balance range and coordinated stability tests, with the sway-meter directed anteriorly. For the maximum balance range test, participants were instructed to lean as far forwards and backwards as possible without bending at the hips and the maximum anteroposterior excursion was recorded. The coordinated stability test required the participants to bend and rotate their body so that the pen on the end of the sway-meter remained within a convoluted track placed on top of an adjustable height table. An error score was calculated by summing the number of occasions that the pen failed to stay within the path. 
To generate an individual's overall falls risk score, web-based software combined an individual's results from the following tests: edge contrast sensitivity, proprioception, quadriceps strength, hand reaction time and sway on foam (eyes open). ${ }^{1}$ These tests have previously been selected from discriminant function analyses and have been shown to discriminate older fallers from non-fallers [14].

\section{Cognition}

The MMSE [16] assessed orientation to time and place, word registration, attention, calculation, recent word recall, language and visual construction at each session. Scores of 27-30, 21-26, 11-20 and 0-10 indicated normal cognition, mild, moderate and severe cognitive impairment, respectively.

\section{Statistical Analysis}

Statistical tests were conducted using SPSS version 13 (SPSS Inc., Chicago, Ill., USA). Distributions of continuously scored variables were analyzed for normality. Variables with skewed distributions were transformed to a logarithmic scale. If data were not transformable, non-parametric equivalent statistics were used. To examine PPA scores for floor or ceiling effects, a frequency table for each baseline PPA variable was generated using SPSS and the percentage of participants scoring the lowest and highest scores was calculated. Raw PPA data were entered into web-based software created for the PPA and adjusted for age and sex and converted to appropriate units.

Test-retest reliability of each PPA variable and the MMSE score were evaluated using the intra-class correlation coefficient (ICC). Additionally, paired t tests compared mean values for each PPA variable and the MMSE over the 2 measurement sessions. ICCs $(3,1)$ were calculated [17]. ICCs were interpreted according to Shrout and Fleiss [17], where ICCs $>0.75,0.40-0.75$ and $<0.40$ correspond to excellent, fair-to-good and poor reliability, respectively. To estimate systematic variation, a series of paired $t$ tests were used.

Differences in PPA scores between AD and control groups were evaluated using a series of independent-samples t tests. To calculate effect sizes, pooled standard deviations were used according to the method for 2 independent groups, and were interpreted as negligible $(0-0.2)$, small $(0.2-0.5)$, medium $(0.5-0.8)$ and large $(>0.8)[18]$. The overall $\alpha$-level was set at $p=0.05$. Finally, Pearson's correlation coefficients were calculated to assess associations between MMSE and PPA scores (averaged across both sessions) in the $\mathrm{AD}$ group.

\section{Results}

\section{Participant Characteristics}

Twenty-nine people with AD were invited to participate; 5 declined and 1 withdrew. Table 1 displays participant characteristics. The prevalence of most self-reported major medical conditions was similar between groups

\footnotetext{
1 www.powmri.edu.au/fbrg/calculator.
}

(table 1). However, a history of cancer and osteoarthritis was more prevalent in controls $(\mathrm{p}<0.05)$. The average number of medications taken by the control group was also greater than that of the AD group.

\section{Feasibility of PPA Tests in People with AD}

Most participants with AD completed PPA tests. However, on at least 1 occasion, 3 were incapable of low-contrast visual acuity, 2 of tactile sensitivity, 1 of ankle dorsiflexion strength, 1 of foot reaction time, 2 of postural sway on foam (eyes open) and 7 of sway on foam (eyes closed). A ceiling effect existed for the contrast sensitivity test as 7 participants (33\%) scored the highest score on both sessions. A floor effect existed for near-tandem stability; $29 \%$ of participants were able to complete the test at baseline, and $14 \%$ completed the test at re-test. Only $19 \%$ of participants completed the test on both occasions.

\section{Test-Retest Reliability of PPA Tests}

Means and standard deviations for PPA scores from both measurement sessions are presented in table 2. Mean values for most PPA scores were similar across both sessions. Paired t tests revealed performance on 2 tests significantly improved from baseline to re-test in people with $\mathrm{AD}$ : hand reaction time $\left(\mathrm{t}_{20}=2.20, \mathrm{p}<0.05\right)$ and maximal balance range $\left(\mathrm{t}_{20}=-2.14, \mathrm{p}<0.05\right)$. However, these differences were small; hand reaction time improved approximately $30 \mathrm{~ms}$ and maximal balance range improved approximately $9 \mathrm{~mm}$ on re-test.

Table 2 displays test-retest reliability data for each PPA variable. ICCs were excellent for high- and low-contrast visual acuity, contrast sensitivity, knee extension strength, coordinated stability and maximal balance range tests, ranging from 0.78 to 0.90 . Fair-to-good ICCs were found for tactile sensitivity, ankle dorsiflexion strength, hand reaction time, sway on foam with eyes closed and the overall falls risk score $(0.43-0.75)$. ICCs were poor for proprioception, foot reaction time, sway on floor (eyes open and closed) and sway on foam with eyes open (0.18$0.39)$.

\section{Test-Retest Reliability of the MMSE}

Overall, the mean MMSE scores were similar on both occasions. A paired t test revealed no significant systematic difference between group baseline and re-test MMSE scores, indicating the group's cognitive function was relatively stable over time. There was no association between MMSE score (averaged across both measurement sessions) and education $(\mathrm{r}=-0.25, \mathrm{p}=0.27)$ or age $(\mathrm{r}=$ 
Table 2. PPA scores for AD cases and controls

\begin{tabular}{|c|c|c|c|c|}
\hline \multirow[t]{2}{*}{ Test } & \multicolumn{3}{|c|}{ AD cases $(n=21)$} & \multirow{2}{*}{$\begin{array}{l}\text { Controls } \\
(\mathrm{n}=21)\end{array}$} \\
\hline & session 1 & session 2 & ICC & \\
\hline Visual acuity - high contrast (MAR) & $1.8(1.0)$ & $1.8(0.9)$ & $0.80[0.57-0.91]$ & $1.5(0.7)$ \\
\hline Visual acuity - low contrast (MAR) & $4.0(1.5)$ & $4.0(1.5)$ & $0.87[0.71-0.95]$ & $3.9(3.7)$ \\
\hline Contrast sensitivity, $\mathrm{dB}$ & $21.1(2.8)$ & $21.6(2.8)$ & $0.86[0.68-0.94]$ & $20.8(2.9)$ \\
\hline Proprioception, ${ }^{\circ}$ & $1.9(1.4)$ & $1.8(1.1)$ & $0.18[-0.26$ to 0.56$]$ & $1.5(1.1)$ \\
\hline Tactile sensitivity, $\log _{10} \mathrm{mg}$ & $4.7(0.6)$ & $4.8(0.7)$ & $0.74[0.43-0.89]$ & $4.6(0.5)$ \\
\hline Ankle dorsiflexion strength, $\mathrm{kg}$ & $9.8(5.2)$ & $10.7(5.0)$ & $0.69[0.37-0.87]$ & $11.3(5.5)$ \\
\hline Knee extension strength, kg & $26.1(11.2)$ & $26.3(12.4)$ & $0.78[0.54-0.91]$ & $22.4(7.7)$ \\
\hline Reaction time - hand, ms & $357(89)$ & $328(84)$ & $0.75[0.48-0.89]$ & $251(44)^{* *}$ \\
\hline Reaction time - foot, ms & $379(76)$ & $375(89)$ & $0.39[0.03-0.70]$ & $326(80)^{*}$ \\
\hline Sway floor - eyes open, $\mathrm{mm}$ & $54(22)$ & $48(20)$ & $0.28[-0.16$ to 0.63$]$ & $73(64)$ \\
\hline Sway floor - eyes closed, $\mathrm{mm}$ & $81(9)$ & $81(13)$ & $0.24[-0.22$ to 0.61$]$ & $94(30)$ \\
\hline Sway foam - eyes open, $\mathrm{mm}$ & $134(79)$ & $122(62)$ & $0.33[-0.11$ to 0.66$]$ & $145(70)$ \\
\hline Sway foam - eyes closed, $\mathrm{mm}$ & $399(162)$ & $379(202)$ & $0.43[0.01-0.72]$ & $388(154)$ \\
\hline Coordinated stability, errors & $16.7(10.7)$ & $15.4(10.2)$ & $0.791[0.56-0.91]$ & $9.2(9.1)^{*}$ \\
\hline Maximum balance range, $\mathrm{mm}$ & $133(41)$ & $142(43)$ & $0.90[0.76-0.96]$ & $150(44)$ \\
\hline Overall falls risk score & $1.3(1.2)$ & $1.0(1.1)$ & $0.69[0.37-0.86]$ & $0.6(0.8)^{*}$ \\
\hline
\end{tabular}

Values are means with standard deviations in parentheses. Figures in square brackets represent 95\% CI.

${ }^{*} \mathrm{p}<0.05$ : significant difference between AD cases and controls; ${ }^{* *} \mathrm{p}<0.01$ : significant difference between AD cases and controls.

$0.16, \mathrm{p}=0.50)$. The MMSE demonstrated excellent testretest reliability in $\mathrm{AD}(\mathrm{ICC}=0.78,95 \% \mathrm{CI}=0.53$ to $0.90)$.

\section{Differences in PPA Scores between AD Cases and Controls}

Table 2 displays descriptive statistics for baseline AD and control group PPA scores. AD cases had a significantly slower hand reaction time $\left(\mathrm{t}_{40}=-4.86, \mathrm{p}<0.01\right)$, foot reaction time $\left(\mathrm{t}_{40}=-2.26, \mathrm{p}<0.05\right)$ and worse coordinated stability $\left(\mathrm{t}_{40}=-2.44, \mathrm{p}<0.05\right)$ than controls and exhibited a higher overall falls risk score $\left(\mathrm{t}_{40}=-2.41, \mathrm{p}<\right.$ 0.05). Large effect sizes were found for hand reaction time $(\mathrm{d}=1.50)$, foot reaction time $(\mathrm{d}=1.70)$ and coordinated stability $(d=0.75)$. A medium effect size $(d=0.74)$ was found for the overall falls risk score.

\section{Correlation between MMSE Score and PPA}

Variables in People with AD

PPA and MMSE scores were correlated and analyzed on a scatter plot to determine the association between each PPA variable and cognition (MMSE score). A significant association was found between MMSE and coordinated stability scores $(\mathrm{r}=-0.51, \mathrm{p}<0.05)$, indicating that the greater the degree of cognitive impairment, the

Falls Risk Assessment in Alzheimer's Disease greater the number of errors when performing the coordinated stability test. No other significant associations were found between the PPA scores and the MMSE score.

\section{Discussion}

The aim of this study was to examine the feasibility and test-retest reliability of PPA scores in older people with $\mathrm{AD}$, and to compare PPA performances between $\mathrm{AD}$ cases and controls. Overall, the findings indicate that a physiological approach to falls risk assessment appears appropriate in older people with AD. Most PPA tests had adequate test-retest reliability when tested on 2 occasions, 1 week apart. The PPA tests also appear to discriminate between $\mathrm{AD}$ cases and controls, with significant differences observed for hand and foot reaction time, coordinated stability and the overall falls risk score.

Most participants were capable of completing the PPA tests, suggesting that this test battery is feasible in people with mild to moderate AD. However, the floor effect associated with the near-tandem stability test suggests that it may not be a feasible balance assessment in this population. In a previous study of 156 cognitively intact older 
people [19], all participants completed the test, suggesting that people with AD may have particular difficulty maintaining near-tandem stability. The other PPA tests showed a similar pattern of reliability in people with AD as found previously in the general older population [14]. Test-retest reliability was excellent for vision, knee extension strength and coordinated stability, and fair-to-good for tactile sensitivity and hand reaction time tests. However, proprioception, foot reaction time, ankle dorsiflexion strength and postural sway tests were less reliable in people with AD than the general older population. Several factors may have contributed to this. For example, impaired concentration is a feature of mild to moderate $\mathrm{AD}$ [15] and may have influenced the reliability of the proprioception and foot reaction time tests, which have relatively high concentration requirements. Reliability of the foot reaction time test may also have been influenced by test order, as foot reaction time was completed directly after hand reaction time and the results may have been confounded by mental fatigue.

The tactile sensitivity test was actually more reliable in people with $\mathrm{AD}(\mathrm{ICC}=0.74)$ compared to values previously reported in cognitively intact older people (ICC = 0.51) [14]. However, it is worth noting that many participants demonstrated some difficulty in grasping the concept of the task and needed frequent verbal reminders to complete the test (i.e. to report whether they felt the stimulus on the letter ' $A$ ' or ' $B$ '). Therefore, people who performed poorly in this test at baseline also performed poorly at re-test. Despite the high ICC, the validity of tactile sensitivity testing is uncertain, as the results may have been influenced more by cognition than peripheral sensation.

A significantly slower reaction time in people with $\mathrm{AD}$ complements the findings of Whitney et al. [20], who observed a significantly slower reaction time in older people with cognitive impairment compared to cognitively healthy older people. The significantly higher overall falls risk score observed in the $\mathrm{AD}$ group provides further evidence of an increased risk of falling in older people with AD [2]. The fact that this falls risk score was based on a physiological profile further implicates underlying sensorimotor deficits as risk factors for falls in people with AD [6]. Whitney et al. [20] similarly found that the PPA falls risk score was significantly higher in community-dwelling older people with cognitive impairment (a score $<8$ on the Abbreviated Mental Test) compared with cognitively intact older people. In the present study, although falls risk was significantly higher in people with $\mathrm{AD}$, the group's score was within the normal age-related falls risk range. This may be explained by the fact that the average severity of dementia in the AD group was mild; so it is possible that the group's fall risk would have been higher if people with severe dementia had been included.

No significant association between cognition (MMSE score) and the overall falls risk score was found. This lack of association is consistent with the findings of Camicioli and Licis [6], who found that falling in people with advanced $\mathrm{AD}$ was not related to dementia severity. A possible explanation for this study's finding is that the tests upon which the falls risk score was based (i.e. edge contrast sensitivity, proprioception, quadriceps strength, hand reaction time and sway on foam, eyes open) were not particularly cognitively demanding. However, this finding is inconsistent with Whitney et al. [20], who found cognitive impairment was a significant, independent predictor of a high PPA falls risk score. A likely explanation for this difference is the larger sample size used $(n=110)$ compared to the current study $(n=21)$ and the restricted range of $\mathrm{AD}$ severity in our group.

The significantly worse performances on the coordinated stability test in people with $\mathrm{AD}$ compared to controls may be partially explained by the cognitive demands of this test, which is further substantiated by the observed correlation between coordinated stability and the MMSE score. A significantly slower foot reaction time may also have contributed to worse coordinated stability in people with $\mathrm{AD}$, as foot reaction time has previously been shown to be an independent predictor of coordinated stability test performance [21]. The observation of reduced coordinated stability suggests that people with $\mathrm{AD}$ may be even less stable when undertaking activities requiring voluntary movements than cognitively intact older people [22], and suggests that this test may be a better indicator of balance impairment in people with $\mathrm{AD}$ than tests of postural sway. Indeed, given that the coordinated stability test had excellent test-retest reliability, high feasibility, discriminated between $\mathrm{AD}$ and control groups and reflected poor cognition, it may be particularly valuable in identifying people with $\mathrm{AD}$ who are at risk of falling.

These findings need to be interpreted in the context of the study's limitations. Firstly, the sample size was relatively small and included people with only mild to moderate AD. Therefore, these results may not be generalizable to the broader population of people with AD. Secondly, while deficits in some aspects of physiological function known to be associated with falls have been identified in those with $\mathrm{AD}$, it remains to be seen whether these deficits are significant enough to actually in- 
crease the risk of falls. To address this question, a larger study, in which physiological function is assessed at baseline and falls are prospectively documented, would need to be undertaken.

\section{Conclusion}

Physiological falls risk assessment using the PPA is feasible in older people with mild to moderate AD. Older people with $\mathrm{AD}$ demonstrated significant impairments in several physiological domains, particularly reaction time, compared to cognitively intact controls. The coordinated stability test may be the most beneficial balance test to identify high-risk fallers in older people with $\mathrm{AD}$ due to its high reliability, ability to discriminate between older people with and without $\mathrm{AD}$, and association with MMSE scores. Prospective studies are now required to evaluate the validity of the PPA in predicting falls in people with AD.

\section{Acknowledgements}

Partial support for this project was provided by Alzheimer's Australia. H.B.M. is currently a National Health and Medical Research Council of Australia fellow (Clinical Career Development Award, ID: 433049).

\section{References}

$\checkmark 1$ Buchner D, Larson E: Falls and fractures in patients with Alzheimer-type dementia. JAMA 1987;257:1492-1495.

-2 Morris J, Rubin E, Morris E, Mandel SA: Senile dementia of the Alzheimer's type: an important risk factor for serious falls. J Gerontol 1987;42:412-417.

3 Melton LJ 3rd, Beard CM, Kokmen E, Atkinson EJ, O'Fallon WM: Fracture risk in patients with Alzheimer's disease. J Am Geriatr Soc 1994;42:614-619.

4 Weller I, Schatzker J: Hip fractures and Alzheimer's disease in elderly institutionalized Canadians. Ann Epidemiol 2004;14:319324.

$\checkmark 5$ Horikawa E, Matsui T, Arai H, Seki T, Iwasaki K, Sasaki H: Risk of falls in Alzheimer's disease: a prospective study. Intern Med 2005; 44:717-721.

-6 Camicioli R, Licis L: Motor impairment predicts falls in specialized Alzheimer care units. Alzheimer Dis Assoc Disord 2004;18. 214-218.

7 Uhlmann RF, Larson EB, Koepsell TD, Rees TS, Duckert LG: Visual impairment and cognitive dysfunction in Alzheimer's disease. J Gen Intern Med 1991;6:126-132.

8 Gordon B, Carson K: The basis for choice reaction time slowing in Alzheimer's disease. Brain Cogn 1990;13:148-166.
-9 Manckoundia P, Pfitzenmeyer P, d'Athis P, Dubost V, Mourey F: Impact of cognitive task on the posture of elderly subjects with Alzheimer's disease compared to healthy elderly subjects. Mov Disord 2006;21:236241.

10 Camicioli R, Howieson D, Lehman S, Kaye J: Talking while walking: the effect of a dual task in aging and Alzheimer's disease. Neurology 1997;48:955-958.

11 Webster KE, Merory JR, Wittwer JE: Gait variability in community dwelling adults with Alzheimer disease. Alzheimer Dis Assoc Disord 2006;20:37-40.

12 Moreland JD, Richardson JA, Goldsmith $\mathrm{CH}$, Clase CM: Muscle weakness and falls in older adults: a systematic review and metaanalysis. J Am Geriatr Soc 2004;52:11211129.

13 Rubenstein LZ: Falls in older people: epidemiology, risk factors and strategies for prevention. Age Ageing 2006;35:ii37-ii41.

14 Lord SR, Menz HB, Tiedemann A: A physiological profile approach to falls risk assessment and prevention. Phys Ther 2003;83: 237-252.

- 15 McKhann G, Drachman D, Folstein M, Katzman R, Price D, Stadlan EM: Clinical diagnosis of Alzheimer's disease: report of the NINCDS-ADRDA Work Group under the auspices of Department of Health and Human Services Task Force on Alzheimer's Disease. Neurology 1984;34:939-944.
16 Folstein MF, Folstein SE, McHugh PR: 'Minimental state': a practical method for grading the cognitive state of patients for the clinician. J Psychiatr Res 1975;12:189-198.

17 Shrout PE, Fleiss JL: Intraclass correlations: uses in assessing rater reliability. Psychol Bull 1979;86:420-428.

18 Cohen J: Statistical Power Analysis for the Behavioral Sciences. Hillsdale, Erlbaum, 1988.

19 Lord SR, Rogers MW, Howland A, Fitzpatrick R: Lateral stability, sensorimotor function and falls in older people. J Am Geriatr Soc 1999;47:1077-1081.

20 Whitney JC, Lord SR, Close JC: Streamlining assessment and intervention in a falls clinic using the Timed Up and Go Test and Physiological Profile Assessments. Age Ageing 2005;34:567-571.

21 Menz HB, Morris ME, Lord SR: Foot and ankle characteristics associated with impaired balance and functional ability in older people. J Gerontol A Biol Med Sci 2005;60:15461552.

22 Duncan G, Wilson JA, MacLennan WJ, Lewis S: Clinical correlates of sway in elderly people living at home. Gerontology 1992;38: $160-166$ 